\title{
Predictive model for Photodynamic Therapy with gold nanoparticles as vehicle for the photosensitizer delivery
}

\author{
I. Salas-García, F. Fanjul-Vélez, N. Ortega-Quijano, J. L. Arce-Diego* \\ Applied Optical Techniques Group, TEISA Department, University of Cantabria, Av. de los Castros \\ s/n, 39005 Santander (Spain)
}

\begin{abstract}
Photodynamic Therapy offers multiple advantages to treat nonmelanoma skin cancer compared to conventional treatment techniques such as surgery, radiotherapy or chemotherapy. Among these advantages are particularly relevant its noninvasive nature, the use of non ionizing radiation and its high selectivity. However the therapeutic efficiency of the current clinical protocol is not complete in all the patients and depends on the type of pathology. Emerging strategies to overcome its current shortcomings include the use of nanostructures that can act as carriers for conventional photosensitizers and improve the treatment selectivity and provide a controlled release of the photoactive agent. In this work, a model for photodynamic therapy combined with gold nanocarriers for a photosensitizer commonly used in dermatology is presented and applied to a basal cell carcinoma in order to predict the cytotoxic agent spatial and temporal evolution.
\end{abstract}

Keywords: Photodynamic Therapy, gold nanoparticles, photosensitizer, basal cell carcinoma

\section{INTRODUCTION}

Photodynamic Therapy (PDT) is an optical treatment modality used in several clinical fields to destroy malignant tissues ${ }^{1}$. It consists of the administration of a photosensitive substance known as photosensitizer which is activated by the subsequent optical irradiation of the tumor area. Thus it is based on a photochemical interaction between three essential components: the photosensitizer, the light and the oxygen. As a consequence reactive oxygen species are produced and destroy the cancerous cells. On the other hand the photosensitizer fluorescence allows to use it as contrast agent for fluorescence based imaging, providing PDT with diagnostic functionality used in several applications such as guided resection, treatment planning and monitoring, outcome treatment assessment or the study of the interactions produced during PDT and the mechanisms involved in cell death ${ }^{2}$.

In dermatology, PDT offers multiple advantages to treat nonmelanoma skin cancer compared to conventional treatment techniques such as surgery, radiotherapy or chemotherapy ${ }^{3}$. Among these advantages are particularly relevant its noninvasive nature, the use of non ionizing radiation and its high selectivity. However the therapeutic efficiency of the current clinical protocol is not complete in all the patients and depends on the type of pathology.

Emerging strategies to overcome the current PDT shortcomings related to the photosensitizer accumulation in the target tissue or the optical radiation distribution include the use of nanostructures with conventional PDT. The future potential ability of nanotechnology to enhance the critical issues of PDT and finally improve its therapeutic effectiveness makes essential to assess dosimetric approaches based on the combined use of PDT and nanoparticles. These last ones can act as carriers for conventional photosensitizers, as excitation energy transducers or active photodynamic elements ${ }^{4,5}$. Among the former, gold nanoparticles have demonstrated to be excellent carriers for the photosensitizer delivery ${ }^{6}$. As well as being particularly interesting due to their ease of synthesis and chemical modification or their unique optical properties ${ }^{7}$. The use of nanoparticles as delivery vehicle has a great interest to provide an enhanced treatment selectivity in the target tissue and a controlled release of the photoactive agent. The nanoparticles encapsulating the photosensitizer molecules or with the phtosensitizer covalently attached to the nanoparticles surface, make possible to deliver high payloads of photoactive agent, including hydrophobic agents as well as an improved therapeutic selectivity and accumulation in the tumor tissues owing to the enhanced permeability retention (EPR) effects and antibody conjugation.

*arcedj@unican.es; phone +34 942201545

Medical Laser Applications and Laser-Tissue Interactions VI, edited by Lothar D. Lilge, Ronald Sroka,

Proc. of OSA Biomedical Optics-SPIE Vol. 8803, 88030N · @ 2013 OSA-SPIE

CCC code: $1605-7422 / 13 / \$ 18 \cdot$ doi: $10.1117 / 12.2031959$ 
The future development of customized dosimetry for novel nanoparticle-based PDT requires predictive models able to adjust a great amount of parameters of different nature in order to get an optimal tumor removal ${ }^{8}$. These models must consider the photosensitizer-nanoparticle distribution in the malignant tissue, its influence in the optical radiation distribution and the photochemical reactions underlying PDT in order to get an accurate dosimetric adjustment. Previous studies have theoretically investigated the spatial distribution of nanoparticles by particle trajectory tracking models or its optical and thermal properties, however there is a very limited number of works related to the development of mathematical models that characterize the whole photodynamic process with nanoparticles, which includes not only the photosensitizer-nanoparticle distribution in the malignant tissue but also its influence in the optical radiation distribution and the photochemical reactions evolution. This work is devoted to a predictive model focused on the use of gold nanoparticles as carriers for a photosensitizer commonly used in dermatology. Section 2 describes the model basis and thereafter it is applied to a basal cell carcinoma. The obtained results are presented in Section 3. Finally, the conclusions are listed in the Section 4.

\section{PREDICTIVE MODEL FOR PHOTODYNAMIC THERAPY COMBINED WITH GOLD NANOPARTICLES}

The predictive model for nanoparticle-based PDT takes into account the main processes involved in the photodynamic process as well as those effects produced by the administration of gold nanoparticles in the photosensitizer distribution and the optical propagation.

The nanoparticles transport mechanism in the tumor tissue once they leave the bloodstream through the leaky tumor vasculature is obtained by means of a diffusion model considering the vessel walls as the main barrier to the diffusion into the target tissue. The time dependent distribution of the nanoparticles in the tumor tissue is described by Fick's first law of mass flow in equation (1), which is widely employed to model the transport of solutes in tissues. According to this law the diffusion of nanoparticles occurs from areas of high concentration, the vessel walls in this case, to those where the concentration is lower. In this expression $\vec{j}$ is the flux vector, $N_{T}$ is the concentration of nanoparticles and $D$ is the diffusivity.

$$
\vec{j}=-D \cdot \operatorname{grad} N_{T}
$$

The temporal evolution of the nanoparticles concentration depending on distance from the vessel wall, $x$, can be calculated as':

$$
N_{T}(t)=N_{o} \int_{0}^{t}\left(\frac{K}{\sqrt{D \pi t^{\prime}}} e^{-\frac{x^{2}}{4 D t^{\prime}}}-\frac{K^{2}}{D} e^{\frac{K}{D} x} e^{\frac{K^{2}}{D} t^{\prime}} \operatorname{erfc}\left(\frac{K}{\sqrt{D}} \sqrt{t^{\prime}}+\frac{x}{2 \sqrt{D t^{\prime}}}\right)\right) e^{-\frac{t^{\prime}}{\tau}} d t^{\prime}
$$

where $N_{o}$ is the initial nanoparticles concentration bound to the vessel wall at the beginning of the diffusion time $t=0$, $K$ is the vessel wall permeability and $\tau$ is the particle relaxation time. The photosensitizer concentration at the end of the incubation period is calculated from the nanoparticles distribution profile in the malignant tissue and the photosensitizer payload carried by the nanoparticle.

The optical radiation distribution in the tissue is obtained by means of the Monte Carlo method applied to the Radiation Transport Equation (RTE) $)^{10,11}$, in the expression (3) for a steady-state situation, taking into account the effects of the nanopaticles on the absorption and scattering via Mie Theory ${ }^{12}$. The model assumes that the scattering events are sufficiently numerous as to the light to be considered incoherent, in such a way that polarization or interference effects can be neglected. As a consequence, the basic parameter of light is the specific intensity, $I(r, \hat{s})$. The radiation is expected to be at the point $\vec{r}$, and to follow the direction $\hat{s}$. The scattering events are treated according to the scattering phase function, $p\left(\hat{s} \cdot \hat{s}^{\prime}\right)$, which contains the probabilities of light to be scattered in the different directions. Optical radiation comes from direction $\hat{s}^{\prime}$ and is redirected to $\hat{s}$. According to the differential RTE, the radiation from a particle attenuates due to absorption and scattering and also gains power because another particle can scatter light in the direction of the particle of interest ${ }^{1}$. Thus in (3), the absorption and scattering events are taken into account by means of $\mu_{a}$ and 
$\mu_{s}$ respectively. Both of them include the optical properties of the nanoparticles and the type of pathological tissue where they are embedded.

$$
\hat{s} \cdot \bar{\nabla} I(r, \hat{s})=-\left(\mu_{a}+\mu_{s}\right) I(r, \hat{s})+\frac{\mu_{s}}{4 \pi} \int_{4 \pi} p\left(\hat{s} \cdot \hat{s}^{\prime}\right) I\left(r, \hat{s}^{\prime}\right) d \Omega^{\prime}
$$

The Monte Carlo method has demonstrated its applicability and accuracy, to solve the radiation transport equation compared with exact solutions. In this work the implementation of the Monte Carlo method applied to the RTT model is the one by Wang and Jacques ${ }^{10}$. The Monte Carlo program assumes that the optical beam is infinitely narrow, and that it has perpendicular incidence. The second assumption is reasonable, but the former can provoke serious disappointments with the reality, particularly if the dimensions of the optical spot and the tissue are of the same order. In order to correct this limitation, another program by the same authors ${ }^{11}$ implements the convolution of the results. In this way, the solution of the Monte Carlo analysis can be later transformed for taking into account a cylindrical geometry of the laser beam like the one used in this work. This implementation of the Monte Carlo model is also multi-layered, so it is possible to define several layers of different materials, with their borders always perpendicular to the laser beam, which is very useful in the skin tissues due to they usually can be divided in different strata.

Mie's theory permits to calculate the efficiency factors of scattering, $Q_{\text {sca }}$, and extinction, $Q_{e x t}$, for homogeneous spherical nanoparticles with radius $r$ taking into account the complex refractive index of the material $\mathrm{m}$ by means of the expressions (4) and (5) respectively. Then the efficiency factor of absorption $Q_{a b s}$ can be easily calculated from these last ones as in expression (6).

$$
\begin{gathered}
Q_{s c a}=\frac{2}{x^{2}} \sum_{n=1}^{\infty}(2 n+1)\left(\left|a_{n}\right|^{2}+\left|b_{n}\right|^{2}\right) \\
Q_{e x t}=\frac{2}{x^{2}} \sum_{n=1}^{\infty}(2 n+1) \operatorname{Re}\left(a_{n}+b_{n}\right) \\
Q_{a b s}=Q_{e x t}-Q_{s c a}
\end{gathered}
$$

The amplitude coefficients for the scattered field, $a_{n}$ and $b_{n}$, are calculated as in the expressions (7) and (8), involving spherical Bessel functions ${ }^{13}$. In these expressions, $x=k r$ is the size parameter, $k=2 \pi / \lambda$ is the wave number, $\lambda$ is the wavelength in the ambient medium and $\mu_{1}$ is the ratio of the magnetic permeability of the sphere to the magnetic permeability of the ambient medium.

$$
\begin{gathered}
a_{n}=\frac{m^{2} j_{n}(m x)\left[x j_{n}(x)\right]^{\prime}-\mu_{1} j_{n}(x)\left[m x j_{n}(m x)\right]^{\prime}}{m^{2} j_{n}(m x)\left[x h_{n}^{(1)}(x)\right]^{\prime}-\mu_{1} h_{n}^{(1)}(x)\left[m x j_{n}(m x)\right]^{\prime}} \\
b_{n}=\frac{\mu_{1} j_{n}(m x)\left[x j_{n}(x)\right]^{\prime}-j_{n}(x)\left[m x j_{n}(m x)\right]^{\prime}}{\mu_{1} j_{n}(m x)\left[x h_{n}^{(1)}(x)\right]^{\prime}-h_{n}^{(1)}(x)\left[m x j_{n}(m x)\right]^{\prime}}
\end{gathered}
$$

The efficiency factors previously obtained permit to know the nanoparticles absorption, $\mu_{a N P}$, and scattering, $\mu_{s N P}$, coefficients taking into account the cross section of the spherical nanoparticles and the number of nanoparticles per unit volume as:

$$
\begin{aligned}
& \mu_{a N P}=\pi r^{2} Q_{a b s} N_{T} \\
& \mu_{s N P}=\pi r^{2} Q_{s c a} N_{T}
\end{aligned}
$$

Once the photosensitizer accumulation and light distribution are know, the interaction of both elements with the ground state oxygen via a reactions known as Type II triggers a photochemical reaction responsible for singlet oxygen production ${ }^{14}$. This kind of reactive oxygen is considered as the cytotoxic element in charge of killing the carcinogenic cells. Finally the photochemical reactions that take place during PDT can be modeled by means of a differential 
equations system ${ }^{15,16},(11)$ to (16). This photochemical model is based in the Jablonski diagram for the generation of singlet oxygen in a type II reaction and takes into account the electronic transitions of the molecular components involved in the photodynamic procedure such as the photosensitizer or the oxygen. Its solutions permit to analyze the temporal evolution of the main molecular components concentrations such as the photosensitizer in ground state, $S_{0}$, in singlet excited state, $S_{1}$ and in triplet excited state $T$, the oxygen in ground state, ${ }^{3} O_{2}$, singlet oxygen ${ }^{1} O_{2}$ and the intracellular singlet oxygen receptors, $R$. In these equations, $\tau 1$ is the relaxation time from state $S_{1}$ to $S_{0}, \tau 3$ is the relaxation time from state $T$ to $S_{0}, \tau 0$ is the relaxation time from state ${ }^{1} O_{2}$ to ${ }^{3} O_{2}, \eta_{10}$ is the quantum yield of the transition from state $S_{1}$ to $S_{0}, \eta_{13}$ is the quantum yield of the transition from $S_{1}$ to $T, \eta_{30}$ is the quantum yield of $T$ transition to $S_{0}, \eta_{0}$ is the quantum yield of ${ }^{1} O_{2}$ transition to ${ }^{3} O_{2}, \alpha s$ is the efficiency factor for energy transfer from $T$ to ${ }^{3} \mathrm{O}_{2}, k p b$ stands for the biomolecular photobleaching rate, $k c x$ is the biomolecular cytotoxicity rate, $k s c$ is the rate of reaction of ${ }^{1} O_{2}$ with various oxygen scavengers, $v$ is light speed in tissue, $\rho$ is the photon density present at a point, $\sigma_{p s a}$ is the absorption cross-section of $S_{0}$ molecules, $P$ is the rate of oxygen diffusion and perfusion and $U$ is the cell damage repair rate. The stiff differential equations system was solved by means of a differential equation solver (ode15s) within the Matlab ${ }^{\circledR}$ platform. It was necessary to adjust relative and absolute error tolerances in order to obtain coherent results.

$$
\begin{gathered}
\frac{d\left[S_{0}\right]}{d t}=-v \rho \sigma_{p s a}\left[S_{0}\right]-k p b\left[{ }^{1} O_{2}\right]\left[S_{0}\right]+\frac{\eta_{10}}{\tau 1}\left[S_{1}\right]+\frac{\eta_{30}}{\tau 3}[T]+\frac{\alpha s}{\tau 3}[T]\left[{ }^{3} O_{2}\right] \\
\frac{d S_{1}}{d t}=-\frac{1}{\tau 1}\left[S_{1}\right]+v \rho \sigma_{p s a}\left[S_{0}\right] \\
\frac{d[T]}{d t}=-\frac{\eta_{30}}{\tau 3}[T]-\frac{\alpha s}{\tau 3}[T]\left[{ }^{3} O_{2}\right]+\frac{\eta_{13}}{\tau 1}\left[S_{1}\right] \\
\frac{d\left[{ }^{3} O_{2}\right]}{d t}=-\frac{\alpha s}{\tau 3}[T]\left[{ }^{3} O_{2}\right]+\frac{\eta_{0}}{\tau 0}\left[{ }^{1} O_{2}\right]+P \\
\frac{d\left[{ }^{1} O_{2}\right]}{d t}=-k p b\left[S_{0}\right]\left[{ }^{1} O_{2}\right]-k c x[R]\left[{ }^{1} O_{2}\right]-k s c[C]_{i}\left[{ }^{1} O_{2}\right]-\frac{\eta_{0}}{\tau_{0}}\left[{ }^{1} O_{2}\right]+\frac{\alpha s}{\tau 3}[T]\left[{ }^{3} O_{2}\right] \\
\frac{d[R]}{d t}=-k c x\left[{ }^{1} O_{2}\right][R]+U
\end{gathered}
$$

\section{RESULTS}

The structure of the tissue sample employed corresponds to a basal cell carcinoma of $1 \mathrm{~mm}$ in depth crossed by a blood vessel whereby the particles are diffused in the tumor located at $0.5 \mathrm{~mm}$ from the tumor surface. The diffusion model previously presented allowed us to obtain the time dependent accumulation of nanoparticles bearing the photosensitizer in the tumor tissue once they leave the bloodstream through the leaky tumor vasculature taking into account a vessel permeability of $2.82 \cdot 10^{-7} \mathrm{~cm} / \mathrm{s}$ and a diffusion coefficient of $6.13 \cdot 10^{-7} \mathrm{~cm}^{2} / \mathrm{s}, 9,17$. The initial concentration of nanoparticles in the vessel wall was fixed to $2 \cdot 10^{19} \mathrm{~cm}^{-3}$ and the diffusion time was $3 \mathrm{~h}$. The photoactive agent concentration at the end of the incubation period was calculated from the nanoparticles distribution profile in the malignant tissue assuming a payload of 32 molecules of photosensitizer per nanoparticle.

The optical absorption and scattering properties of $80 \mathrm{~nm}$ gold nanospheres were obtained at the laser wavelength used in PDT $(630-635 \mathrm{~nm})$ with the photosensitizer Methyl Aminolevulinate-Protoporphyrin IX (MAL-PpIX). The optical distribution in the multilayer tissue sample was obtained taking into account the tissue optical properties ${ }^{18,19}$ summarized in Table 1 and those of the nanoparticles for a cylindrical optical beam perpendicular to the tissue sample and an 
irradiance of $100 \mathrm{~mW} / \mathrm{cm}^{2}$. In this case the tumor tissue layer corresponds to a basal cell carcinoma. Figure 1 shows the efficiency factors of scattering, extinction and absorption obtained by means of Mie's theory for the gold nanoparticles employed.

Table 1. Tissue sample optical properties at $635 \mathrm{~nm}\left(\mu_{\mathrm{a}}:\right.$ absorption coefficient, $\mu_{\mathrm{s}}:$ scattering coefficient, g: anisotropy of scattering, $n$ : refractive index)

\begin{tabular}{|c|c|c|c|}
\hline Tissue layer & $\boldsymbol{\mu}_{\mathbf{a}}\left(\mathbf{c m}^{-\mathbf{1}}\right)$ & $\boldsymbol{\mu}_{\mathbf{s}}\left(\mathbf{c m}^{-\mathbf{1}}\right)$ & $\mathbf{g}$ \\
\hline Tumor & 1.5 & 104.76 & 0.79 \\
\hline Vessel wall & 6 & 414 & 0.91 \\
\hline Blood & 25 & 400 & 0.98 \\
\hline
\end{tabular}

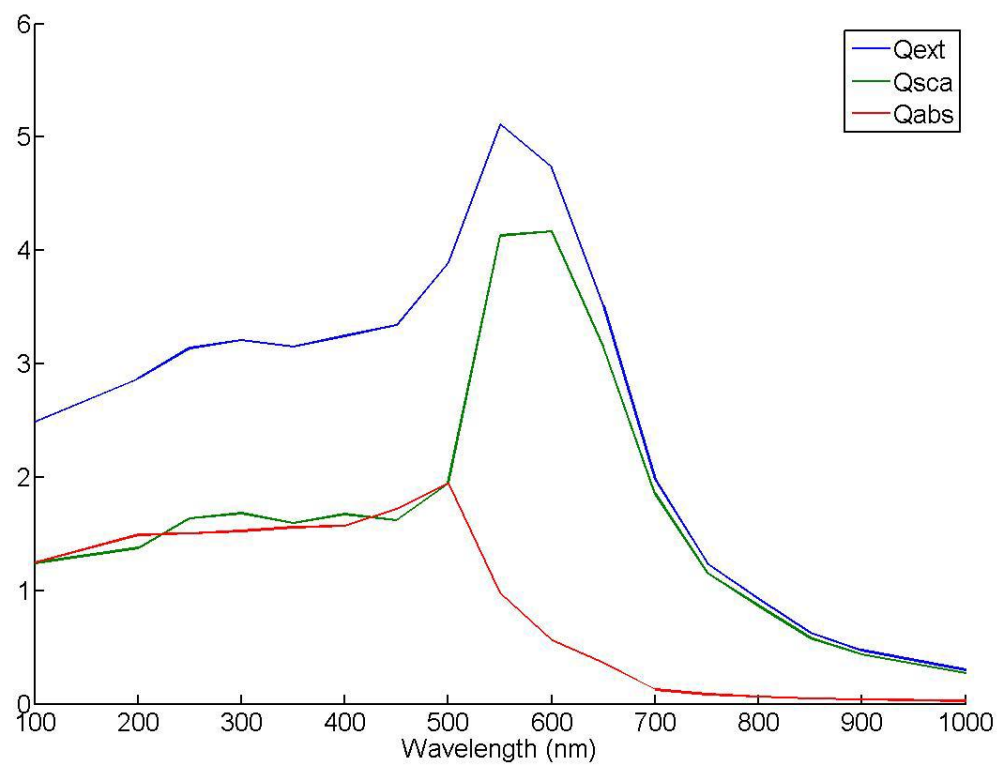

Figure 1. Efficiency factors of scattering (Qsca), extinction (Qext) and absorption (Qabs) for gold nanoparticles.

Literature related to well known photosensitizers of the porphyrins family was explored to assign the parameters values related to the photosensitizer PpIX when they were not available ${ }^{20}$. Thus the absorption cross-section of the PpIX molecule at $635 \mathrm{~nm}$ was derived from a study ${ }^{21}$ of the cellular photosensitizing properties of PpIX carried out in a transformed murine keratinocyte cell line and set to $0.37 \cdot 10^{-15} \mathrm{~cm}^{2}$. The relaxation time from singlet excited state to ground state was set to $7.4 \mathrm{~ns}$ as reported earlier from fluorescence measurements in cells incubated with 5aminolevulinic acid induced PpIX ${ }^{22}$. The triplet state lifetime in vivo ${ }^{23}$ in skin was set to $26 \mu$ s and the relaxation time of singlet oxygen ${ }^{23}$ to its ground state to $0.04 \mu$ s. Quantum yield transitions between different energetic states were adopted to be similar to those previously considered for the photosensitizer Photofrin ${ }^{\circledR} \eta 10=0.2, \eta 30=0.3, \eta 0=0.3$ and $\eta 13=$ 0.8 as well as biomolecular photobleaching, citotoxicity and scavenging rates that were set to $2 \cdot 10^{-10} \mathrm{~cm}^{3} \mathrm{~s}^{-1}, 2 \cdot 10^{-9} \mathrm{~cm}^{3} \mathrm{~s}^{-1}$ and $1 \cdot 10^{-9} \mathrm{~cm}^{3} \mathrm{~s}^{-1}$, respectively ${ }^{16}$. The initial concentration of $\mathrm{S} 1$, T and singlet oxygen is $0 \mathrm{~cm}^{-3}$ due to this kind of molecules are not present in the tissue when the optical irradiation period starts. The tissue sample was supposed to be homogeneously oxygenated and without oxygen supply limitations. The initial concentration of cellular oxygen was set to $5 \cdot 10^{17} \mathrm{~cm}^{-3}$, and diffusion and perfusion rates ${ }^{16}$ to $1 \cdot 10^{12} \mathrm{~cm}^{-3} \mathrm{~s}^{-1}$. The initial concentration of intracellular molecular singlet oxygen receptors was $5 \cdot 10^{17} \mathrm{~cm}^{-3}$, the scavenger concentration was $1 \cdot 10^{3} \mathrm{~cm}^{-3}$ and the cell damage repair rate ${ }^{16}$ $2.6 \cdot 10^{12} \mathrm{~cm}^{-3} \mathrm{~s}^{-1}$.

The photochemical model was employed to obtain the evolution of the different molecular components involved in the photochemical reactions during the treatment progression. Figure 2 shows the singlet oxygen concentration produced in 
the tumor tissue as a function of the distance to the tumor surface and to the center of the optical beam as the therapy progresses. Attending to the results, it can be clearly observed a preferential accumulation of singlet oxygen in those zones closer to the nanoparticles supplier vessel. Therefore the therapy effects related to the cytotoxic action of this reactive oxygen would be more pronounced in the tumor areas closer to the nanoparticles supply (i.e. the vessel for systemic administration) and those types of nonmelanoma skin cancers highly vascularized would be more susceptible to suffer singlet oxygen mediated destruction.

Regarding to the temporal singlet oxygen production, Figure 2 presents the singlet oxygen concentration $\left(\mathrm{cm}^{-3}\right)$ as a function of the distance to the tumor surface, $\mathrm{z}$, and the distance to the center of the cylindrical optical beam, $r$, during the therapy. At $1 \cdot 10^{-24}$ s, $6 \mathrm{~s}, 300 \mathrm{~s}$ and $600 \mathrm{~s}$ in Figure $\left.\left.\left.2 \mathrm{a}\right), \mathrm{b}\right), \mathrm{c}\right)$ and d) respectively.
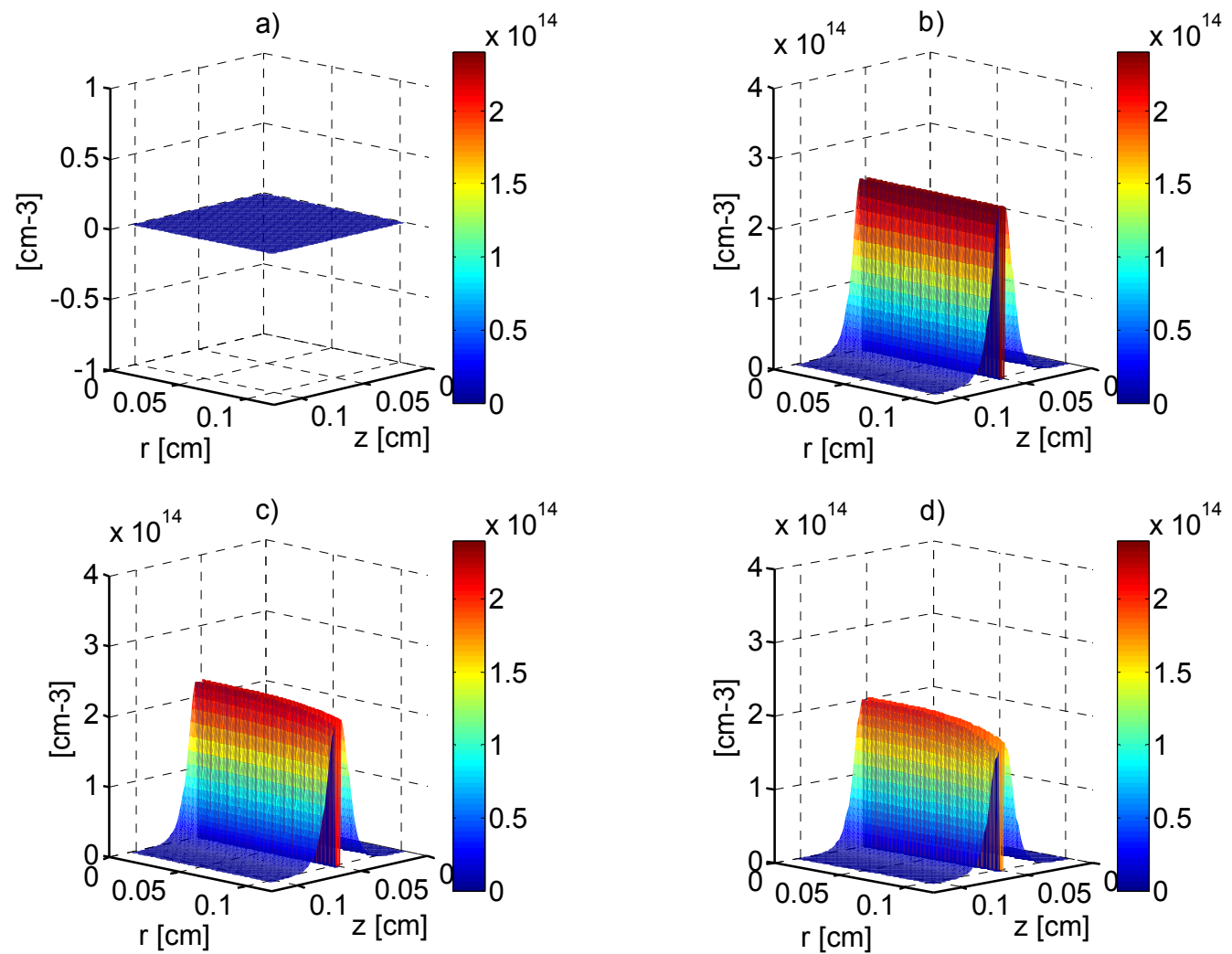

Figure 2. Singlet oxygen concentration $\left(\mathrm{cm}^{-3}\right)$ as a function of cylindrical coordinates ( $\mathrm{z}$ : distance to the tumor surface and $\mathrm{r}$ is the distance to the center of the cylindrical optical beam perpendicular to the tissue sample) at a) $1 \cdot 10^{-24} \mathrm{~s}$, b) $\left.6 \mathrm{~s}, \mathrm{c}\right) 300 \mathrm{~s}$ and d) $600 \mathrm{~s}$ during the therapy.

As it can be observed in Fig. 2 a), singlet oxygen has not yet been produced just when the irradiation starts. However shortly after (Fig. 2 b)) it reaches a high concentration preferentially in those areas near the vessel walls that will progressively decay faster in those points far from the center of the optical beam. Those tumor areas where the production of singlet oxygen is not enough to destroy the malignant tissue will persist after the therapy. Therefore the results showed could help in a future to choose the proper treatment parameters with the purpose of maximize the cytotoxic agent production and consequently the malignant tissue removal. However these results must be interpreted carefully due to the great amount of parameters of different nature involved in the photodynamic process and the large variability between biological media.

\section{CONCLUSIONS}

Despite of the multiple advantages of PDT to treat nonmelanoma skin cancer such as its noninvasive nature, the use of non ionizing radiation or its high selectivity, the therapeutic efficiency of the current clinical protocol is not complete in all the patients and depends on the type of pathology. Emerging strategies to overcome the current PDT shortcomings 
include the use of nanotechnology combined with conventional PDT. The future potential ability of nanotechnology to enhance the critical issues of PDT and improve its therapeutic effectiveness makes essential to assess dosimetric approaches based on the combined use of PDT and nanoparticles. These last ones can assume different roles in the photodynamic process as carriers for conventional photosensitizers, excitation energy transducers or active photodynamic elements. Gold nanoparticles have unique optical properties, are easy to synthesize and have demonstrated to be excellent carriers for the photosensitizer delivery.

Together with the development of customized dosimetry for novel nanoparticle-based therapeutic strategies, the use of predictive models is essential to optimize the adjustment of the great amount of parameters involved in the final treatment result. A first approach to model the temporal photodynamic response of a basal cell carcinoma using gold nanoparticles as delivery vehicle for a photosensitizer commonly used in dermatology was presented. The distribution of the injected nanocarriers from the vessel wall was characterized by means of Fick's law which permits to know the spatial photosensitizer accumulation from the vascular supply. The efficiency factors of absorption, scattering and extinction were calculated by means of Mie's theory for homogeneous spherical nanoparticles and the complex refractive index of gold. The propagation of light in the tumor tissue with gold nanoparticles embedded was obtained by a numerical Monte Carlo method. Finally a photochemical model provided the temporal evolution of the molecular components involved in the photodynamic process.

The results provide the temporal and spatial evolution of the cytotoxic agent produced during the photochemical reactions when the photosensitizer is delivered bound to gold nanoparticles injected in the bloodstream. The citotoxic agent produced is crucial to predict the photodynamic treatment response with nanoparticles due to its direct relation with the tumor necrosis. It was observed a preferential accumulation of singlet oxygen in those zones closer to the nanoparticles supplier vessel. Therefore the therapy effects related to the cytotoxic action of this reactive oxygen could be expected to be more pronounced in the tumor areas closer to the nanoparticles supply which indicates that those types of nonmelanoma skin cancers highly vascularized would be more susceptible to suffer singlet oxygen mediated destruction for a systemic administration of nanoparticles. Future works will consider a more complex tumor vasculature which matches closely with the specific characteristics of the target tumor.

\section{ACKNOWLEDGEMENTS}

This work has been partially supported by the project MAT2012-38664-C02-01 of the Spanish Ministery of Economy and Competitivenss and by San Cándido Foundation

\section{REFERENCES}

[1] Vo-Dinh, T., [Biomedical Photonics Handbook], CRC Press, Boca Raton, (2003).

[2] Celli, J. P., Spring, B. Q., Rizvi, I., Evans, C. L., Samkoe, K. S., Verma, S., Pogue, B. W., Hasan, T., "Imaging and Photodynamic Therapy: Mechanisms, Monitoring, and Optimization" Chem. Rev. 110, 2795-2838 (2010).

[3] Choudhary, S., Nouri, K., Elsaie, M. L., "Photodynamic therapy in dermatology: a review" Lasers Med Sci 24, 971-980 (2009).

[4] Hamblin, M. R. and Mróz, P., [Advances in Photodynamic Therapy. Basic, Translational and Clinical], Engineering in medicine \& Biology, (2008).

[5] Bechet, D., Couleaud, P., Frochot, C., Viriot, M. L., Guillemin, F., Barberi-Heyob, M., "Nanoparticles as vehicles for delivery of photodynamic therapy agents" Trends in Biotechnology 26, 612-621 (2008).

[6] Wieder, M. E., Hone, D. C., Cook, M. J., Handsley, M. M., Gavrilovic, J., Russell, D. A., "Intracellular photodynamic therapy with photosensitizer-nanoparticle conjugates: cancer therapy using a "Trojan horse" Photochem. Photobiol. Sci. 5, 727-734 (2006).

[7] Feldheim, D. L., Foss, C. A., [Metal Nanoparticles Synthesis, Characterization, and Applications], Marcel Dekker, Inc., New York (2002).

[8] Salas-García, I., Fanjul-Vélez, F. and Arce-Diego, J. L., "Photosensitizer absorption coefficient modeling and necrosis prediction during Photodynamic Therapy" Journal of Photochemistry and Photobiology B-Biology 114, 79-86 (2012). 
[9] Svaasand, L. O., Wyss, P., Wyss, M. T., Tadir, Y., Tromberg, B. J. and Berns, M. W., "Dosimetry model for Photodynamic Therapy with topically administered photosensitizers" Lasers in Surgery and Medicine 18, 139149 (1996).

[10] Wang, L., Jacques, S. L. and Zheng, L., "MCML - Monte Carlo modeling of light transport in multi-layered tissues" Computer methods and programs in biomedicine 47, 131-146 (1995).

[11] Wang, L., Jacques, S. L. and Zheng, L., "CONV - Convolution for responses to a finite diameter photon beam incident on multi-layered tissues", Computer methods and programs in biomedicine 54, 141-150 (1997).

[12] Bohren, C. F. and Huffman, D. R., Absorption and Scattering of Light by Small Particles (John Wiley \& Sons, Inc., 1983).

[13] Mätzler, C., "MATLAB Functions for Mie Scattering and Absorption”, Universitas Bernensis, (2002).

[14] Calzavara-Pinton, P., Venturini, M. and Sala, R., "Photodynamic therapy: update 2006 Part 1: Photochemistry and photobiology" Journal of the European Academy of Dermatology and Venereology 21, 293-302 (2006).

[15] Foster, T. H., Murant, R. S., Bryant, R. G., Knox, R. S., Gibson, S. L. and Hilf, R., "Oxygen Consumption and Diffusion Effects in PDT" Radiation Research 126 (3), 296-303 (1991).

[16] Hu, X. H., Feng, Y., Lu, J. Q., Allison, R. R., Cuenca, R. E., Downie, G. H. and Sibata, C. H., "Modeling of a type II Photofrin-mediated PDT process in a heterogeneous tissue phantom" Photochemistry and Photobiology 81, 1460-1468 (2005).

[17] Yuan, F., Dellian, M., Fukumura, D., Leunig, M., Berk, D. A., Torchilin, V. P. and Jain, R. K., "Vascular Permeability in a Human Tumor Xenograft: Molecular Size Dependence and Cutoff Size" Cancer Research 55, 3752-3756 (1995).

[18] Salomatina, E., Jiang, B., Novak, J. and Yaroslavsky, A. N., "Optical properties of normal and cancerous human skin in the visible and near-infrared spectral range" Journal of Biomedical Optics 11(6), 0640261-9 (2006).

[19] Fanjul-Vélez, F., Romanov, O. G., Arce Diego, J. L., "Efficient 3D numerical approach for temperature prediction in laser irradiated biological tissues" Computers in Biology and Medicine 39, 810-817 (2009).

[20] O'Connor, A. E., Gallagher, W.M., Byrne, A.T. "Porphyrin and nonporphyrin photosensitizers in oncology: preclinical and clinical advances in photodynamic therapy" Photochemistry and Photobiology 85, 1053- 1074 (2009).

[21] Theodossiou, T., MacRobert, A. J., "Comparison of the photodynamic effect of exogenous photoprotoporphyrin and protoporphyrin IX on PAM 212 murine keratinocytes" Photochemistry and Photobiology 76, 530-537 (2002).

[22] Kress, M., Meier, T., Steiner, R., Dolp, F., Erdmann, R., Ortmann, U., Rück, A., "Time resolved microspectrofluorometry and fluorescence lifetime imaging of photosensitizers using picosecond pulsed diode lasers in laser scanning microscopes" Journal of Biomedical Optics 8, 26-32 (2003).

[23] Niedre, M., Patterson, M. S., Wilson, B.C., "Direct near-infrared luminescence detection of singlet oxygen generated by photodynamic therapy in cells in vitro and tissues in vivo" Photochemistry and Photobiology 75, 382-391 (2002). 